\title{
Experimental Analysis on the Counter-Current Dumitrescu- Taylor Bubble Flow in a Smooth Vertical Conduct of Small Diameter
}

\author{
S. Benattalah ${ }^{1}$, F. Aloui ${ }^{2,3,4 \dagger}$ and M. Souhar ${ }^{5}$ \\ ${ }^{1}$ Université Mentouri, Faculté des Sciences, Département de Physique, Laboratoire de Physique Énergétique \\ Ain El Bey 25000, Constantine, Algérie \\ ${ }^{2}$ Université de Nantes, Faculté des Sciences et des Techniques, Département de Physique, \\ 2, rue de la Houssinière BP 92208 - 44322 Nantes Cedex 03, France \\ ${ }^{3}$ GEPEA, CNRS-UMR 6144, DSEE, École des Mines, 4 rue Alfred Kastler - BP20722 - 44307 Nantes Cedex, France \\ ${ }^{4}$ Université de Valenciennes et du Hainaut Cambrésis, TEMPO, EA4542, \\ Le Mont Houy, 59313 Valenciennes Cedex 09- France \\ ${ }^{5}$ ENSEM-INPL, LEMTA (CNRS UMR 875) - 2, avenue de la forêt de haye54516, Vandoeuvre-Les-Nancy, France \\ $\dagger$ Corresponding Author Email: Fethi.Aloui@univ-nantes.fr
}

(Received August 19, 2008; accepted August 25, 2010)

\begin{abstract}
In this paper, an experimental investigation on the Dumitrescu-Taylor bubble in counter-current laminar downward flow in vertical pipe of a small internal diameter pipe is presented. The experimental design is realized to work for low and stable liquid flow rates. The Dumitrescu-Taylor bubble may be stationary or can be in motion with an ascending or descending velocity, and this displacement depends on the downward liquid flow rates. Consequently, the advantage of this device is to carry out the measurements of the velocities inside the gas Dumitrescu-Taylor bubble by Laser Doppler Velocimetry (LDV). Starting from the visual observations and image acquisitions with a fast camera, a qualitative description was brought on the hydrodynamic behavior of the liquid film and the ripples created at the bottom of the Dumitrescu-Taylor bubble. The experimental results show a presence of a long toroïdal vortex inside the gas bubble. It should be noted that previous work using a hot wire does not show the existence of this vortex. Additionally, other hydrodynamic magnitudes were measured as the liquid film thickness, the Dumitrescu-Taylor bubble rising velocity as well as the erosion bubble. Detailed descriptions are brought concerned this erosion. Strange phenomena have been observed primarily ahead of the nose of bubble and on the side of its end.
\end{abstract}

Keywords: Two-phase flow, Experimental study, Taylor bubble, Counter-current laminar flow, Velocity profiles inside the gas Taylor bubble, Film thickness, Rising velocity, Ripples.

\section{NOMENCLATURE}

$D \quad$ inner diameter of the tube (mm)

$e \quad$ liquid film thickness $(\mathrm{mm})$

$E o=\rho_{\ell} g D^{2} / \sigma_{\ell}$ Eötvös number

$F_{r}=U_{T b} / \sqrt{g D}$ Froude number

$g$ gravitational acceleration $\left(\mathrm{m} . \mathrm{s}^{-2}\right)$

$L_{D T b}, L_{b}$ length of the Dumitrescu Taylor bubble (mm)

$M o=g \mu_{\ell}^{4} / \rho_{\ell} \sigma_{\ell}^{3}$ Morton number

$P \quad$ pressure $(\mathrm{Pa})$

$Q_{g}$ gas flowrate $\left(\mathrm{m}^{3} \cdot \mathrm{s}^{-1}\right)$

$Q_{\ell} \quad$ liquid flow rate $\left(\mathrm{m}^{3} . \mathrm{s}^{-1}\right)$

$R \quad$ inner radius of tube ( $\mathrm{mm})$

$r$ radial distance from the center of the tube $(\mathrm{mm})$

Re Reynolds number
$\operatorname{Re}_{U L}$ Reynolds number of the flowing liquid $U_{g c}$ gas velocity at the center of the pipe $\left(\mathrm{m} \cdot \mathrm{s}^{-1}\right)$

$U_{g i}$ gas velocity at gas-liquid interface $\left(\mathrm{m} . \mathrm{s}^{-1}\right)$

$U_{\ell} \quad$ mean downward liquid velocity $\left(\mathrm{m} \cdot \mathrm{s}^{-1}\right)$

$U_{D T b}$ rising Dumitrescu-Taylor bubble velocity $\left(\mathrm{m} \cdot \mathrm{s}^{-1}\right)$

$\tau_{i g}$ gas shear stress at gas-liquid interface $(\mathrm{Pa})$

$\lambda$ wavelength of ripples ( $\mathrm{mm}$ )

$\mu_{g}$ dynamic viscosity of gas (Pa.s)

$\rho_{\ell}, \rho_{g}$ liquid and gas density $\left(\mathrm{kg} . \mathrm{m}^{-3}\right)$

$v_{\ell}$ kinematic viscosity of liquid $\left(\mathrm{m}^{2} . \mathrm{s}^{-1}\right)$

$\sigma$ surface tension $\left(\mathrm{N} . \mathrm{m}^{-1}\right)$ 


\section{INTRODUCTION}

When gas and liquid flow simultaneously in a pipe, a variety of configurations (dispersed, annular and transition flow) related to the spatial distribution of the two phases can be observed. These configurations termed flow patterns can be recognized by the flow maps Mandhane (1956). The one most configuration frequently occurs over certain ranges of flow rates is slug flow. This flow is encountered in large industrial processes as such oil and gas wells, gas-liquid pipeline reactors and process vaporizers. It is a complicated flow patterns as reported by Fernandes and Dukler (1983), Orell and Rembrand (1986). It is characterized by a quasi-periodic alteration of long bubbles and liquid slugs. In vertical flow and at low liquid viscosity, the Dumitrescu-Taylor bubble Clanet et al. (2004) has generally spherical nose and flat bottom. It fills most the pipe cross section and around it, the liquid moves in the form of a film. The Dumitrescu-Taylor bubble has been the subject of numerous studies Dumitrescu (1943), Taylor (1950), Collins (1978), Mao and Dukler (1990), Polonsky et al. (1999), Nogueira et al. (2006) etc. Generally, most of these studies were done in stagnant and co-current vertical flows; few of them were conducted in counter current vertical flows in small tube Griffith et al. (1961), Nicklin (1962), and Martin (1976). The comprehension of the bubbles behaviour in flow is important to understand the general phenomenon of gas-liquid flow. So, the flow around the individual bubble remains always a very attractive subject of research and any additional contribution of experimental data is useful for the progression of the physical model development.

In the present work, we are not going to describe previous work in any consecutive manner. We will rather present our own experimental results obtained on the single Dumitrescu-Taylor bubble rising in the counter-current laminar liquid flow in a vertical cylindrical pipe of small diameter. We recall that the Dumitrescu-Taylor bubble has received several studies which concern it bubble velocity, the hydrodynamics of liquid film around it, the flow behind it bottom and the flow velocity fields inside it (gas). At the beginning, the works are leaning to study the motion of single bubble in stagnant liquid Dumitrescu (1943) and Davies and Taylor (1950). They have arrived by assuming an inviscid flow around the bubble nose, to determine an expression of bubble velocity: $U_{D T b}=C_{1} \sqrt{g D}$, where $U_{D T b}$ is the rise velocity of the Dumitrescu-Taylor bubble in stagnant liquid, the coefficient $C_{1}$ is the Froude number which takes the values 0,351 according to Dumitrescu (1943) or 0,328 according to Davies and Taylor (1950). After, others works are followed by holding the influence of viscosity and surface tension on bubble velocity. White and Beardmore (1962) from their experimental investigation, they have introduced non-dimensional parameters Eötvös number $E o=\rho_{l} g D^{2} / \sigma_{\ell}$ and
$M o=g \mu_{\ell}^{4} / \rho_{\ell} \sigma_{\ell}{ }^{3}$ and have established a comprehensive graphical correlation of Froude number as function of these non-dimensional parameters. When a liquid flows through a tube, the motion of the long bubbles results from the complex influence of both buoyancy and mean motion of the liquid. In upwards flow, the motion of the bubble has been studied by several authors Griffith et al. (1961), Nicklin et al. (1962), Collins (1978), Bendiksen (1985) while in downward flow few studies are carried out Griffith et al. (1961), Nicklin et al. (1962) and Martin et al. (1976). The study of the hydrodynamics of liquid film surrounding the Dumitrescu-Taylor bubble have required various works both experimental and numerical, Goldsmith et al. (1962), Brown (1965), Mao and Dukler (1990), Polonsky (1999), Van Hout (2002), Nogueira et al. (2006) and Bugg et al. (1998). For the flow which occurs behind the bottom of the Dumitrescu-Taylor bubble, one can quote works of Campos (1988) and those of Pinto (1996), and very few studies have been concerned with the field of flow inside the Taylor bubble. The first visualization of the flow velocity fields inside the gas Dumitrescu-Taylor bubble, was carried out by Filla (1976), by forming a white smoke inside the Dumitrescu-Taylor bubble using a simple chemical reaction. The method consists to form an Ammonia $\mathrm{NH}_{3}$ bubble which will cross a column of liquid made up of two immiscible layers. The first layer is carbon tetrachloride enough deep to give to the bubble the possibility to reach the limit velocity. The second is a chloridric acid solution HCL. The emergence of the $\mathrm{NH}_{3}$ bubble into the acid leads to form an Ammonium Chloride fog near the interface which was swept down to the bottom of the bubble. Soon, after the chemical reaction, a downward motion near the interface was clearly shown, indicating a toroïdal vortex. When the diameter is small, this method allows only to see furtive vortex but can not obtain quantitative velocity profiles as for the first measurements of gas velocities within a DumitrescuTaylor bubble which were realized by Eccles (1972) and Rates (1972), by using a miniature hot wire anemometer. The Dumitrescu-Taylor bubble is held stationary by a counter-current flow in a $27 \mathrm{~mm}$ diameter tube. The measurements were carried out in the upper hemispherical portion of the bubble $(\mathrm{L} / \mathrm{D}<2.6)$ with a hot wire which doesn't enable to detect the flow direction. It is seen that these results are in excellent agreement with the theoretical predictions. It is noted also that the theoretical predictions and experimental measurements have divergences at the level velocities in the center, and those inside the interface of gas/liquid.

The purpose of this paper is to report measurements obtained on Dumitrescu-Taylor bubble rising in a vertical duct, in a counter-current flow. These measurements are related to the rising bubble velocity in a counter current flow inside a cylindrical duct where very few experimental works are available (Griffith $e t$ al. 1961; Nicklin et al. 1962; Martin et al. 1976), the velocities profiles inside the bubble and the liquid film thickness around the bubble. Also, a description of the Dumitrescu-Taylor bubble surface quality from visual observations and recordings are presented. The design of the experimental equipment was motivated by the need to work with low and stable liquid flow rates, through a small internal diameter pipe. 


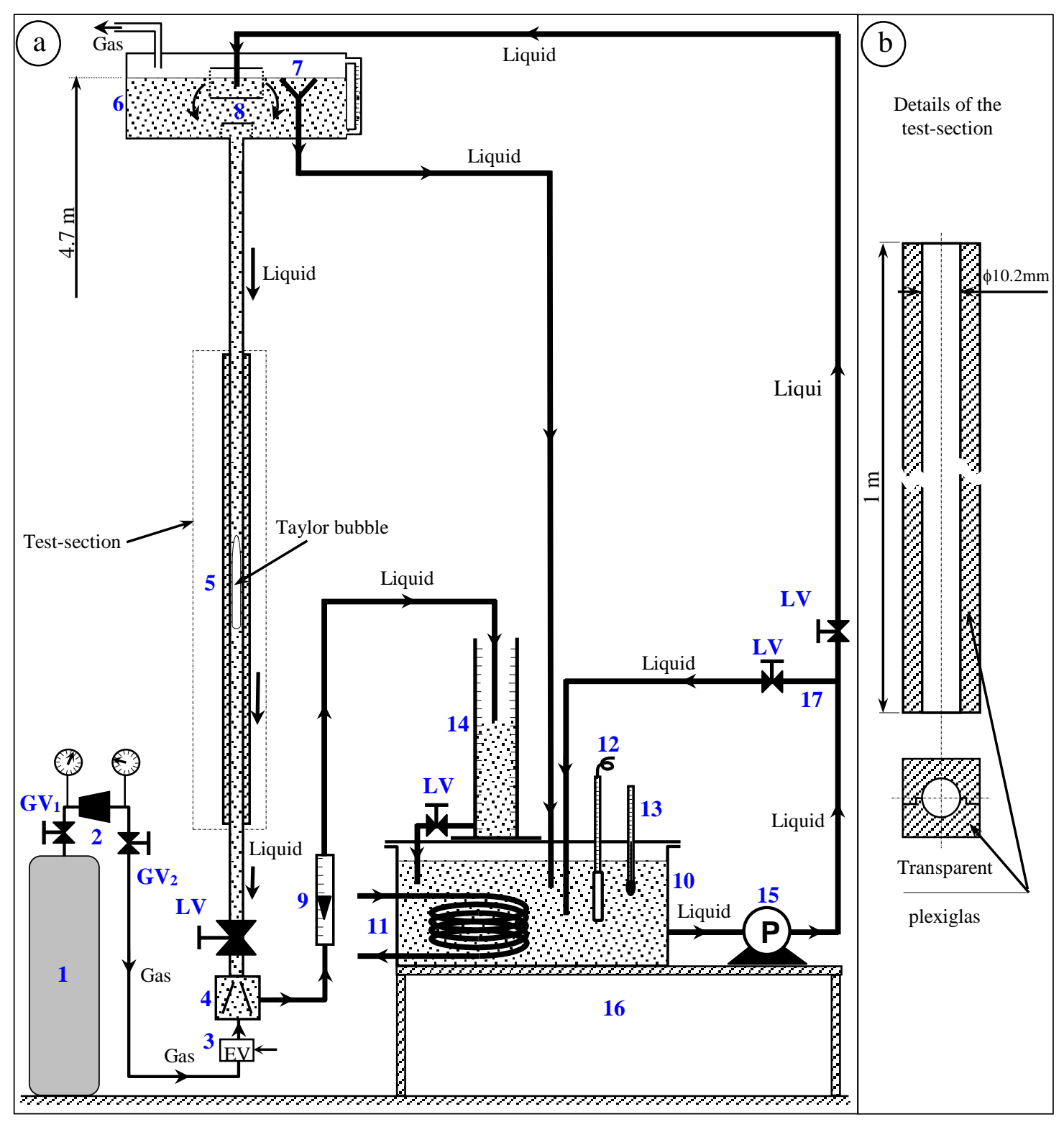

Fig. 1. a) Experimental device: 1. Nitrogen bottle. 2. Pressure reducers. 3. Electro valve. 4. Injector. 5. Test section. 6. Storage tank. 7. Funnel. 8. Tubes. 9. Rotameter. 10. Liquid tank. 11. Heat exchanger. 12. Thermostat. 13.

Thermometer. 14. Graduated tube for liquid flowrate measurements. 15. Pump.16. Support. 17. By-pass. GV. Gas valve. GL. Liquid valve; b) Details of the test-section.

Consequently, (i) we have the possibility to hold a Taylor bubble stationary or to move it with a determined ascending or descending velocity, in downward flows with a uniform velocity profile, (ii) in our case, for all the range of used flow rates, the shape of the bubble doesn't change as the magnitude of the downward flow is increased, which is not the case for the low diameter pipe used by Martin (1962), (iii) The possibility to have a long undisturbed liquid film zone below the Dumitrescu-Taylor bubble nose for make from that zone the velocities measurements inside the bubble, because there, the application of LDA is possible and it becomes difficult to be applied outside this region for the curved optical interfaces.

Motivated by the above considerations, we have designed an experimental device which is described below.

\section{EXPERIMENTAL APPARATUS AND Procedures}

\subsection{Experimental Device}

The experimental apparatus shown in Fig. 1a, is constructed to produce a downward flow with low and stable liquid flow rates, through a small internal diameter pipe. This installation is made of two circuits: a closed liquid circuit maintained at $20 \pm 0.5^{\circ} \mathrm{C}$, and an open gas circuit. The long test channel of $4.7 \mathrm{~m}$ in length is formed with elements of PMMA pipe of $10.2 \mathrm{~mm}$ in inner diameter. It includes a main measuring element of $1 \mathrm{~m}$ in long (Fig. 1b), located at a distance equal to 53 diameters from the gas injector. This element is made in transparent plexiglas, which is practical to use for the Laser Doppler Anemometer 
(LDA) technique application. The liquid circulation is ensured by a pump. The installation has a by-pass in order to correctly regulate the liquid flowrates. The gas is introduced in the injector which is localized at the bottom of the setup. In the inlet of injector, an electrovalve is used to control and calibrate the DumitrescuTaylor bubble. The low flowrate was measured with a rotameter. Other direct measurements were used, in order to well verify the imposed liquid flowrates.

The liquid is an aqueous solution of ferri-ferro-cyanide of potassium. At $20^{\circ} \mathrm{C}$, its physical properties are:

$\rho_{\ell}=1008 \mathrm{~kg} . \mathrm{m}^{-3}, \quad v_{\ell}=0.9910^{-3} \mathrm{~m}^{2} \cdot \mathrm{s}^{-1}, \quad$ and $\sigma=0.070 \mathrm{~N} . \mathrm{m}^{-1}$. The used gas is the nitrogen $\left(\mathrm{N}_{2}\right)$. Its physical properties under the experimental conditions $\quad\left(P=36610^{3} \mathrm{~Pa}, \quad t=20^{\circ} \mathrm{C}\right)$ are: $\rho_{g}=1.56 \mathrm{~kg} . \mathrm{m}^{-3}$, and $v_{g}=11.15 \mathrm{~m}^{2} \mathrm{~s}^{-1}$.

\subsection{Procedure}

\subsubsection{Laser Doppler Velocity Measurements}

To measure the axial velocity component in the gas phase, the Laser Doppler Anemometry was used. It is a measurement technique which has the advantage of not disturbing the flow and to relatively allow measurements close to the gas-liquid interface.

The Dantec Laser Doppler System was used. Two beams from a He-Ne laser were crossed to produce a measurement volume of about $0.009 \mathrm{~mm}^{3}$ (Durrani and Greated, 1977). Two laser beams which are reflected by small particles are used in the flow, passing through the measuring volume of the LDA are received by a photodetector. A beat frequency contained in the reflected beams is used to determine the velocity. In our case, the particles used in the liquid are polystyrenes particles with diameters from 20 to $50 \mu \mathrm{m}$. For the smoke particles used in the gas, their diameters are 0.1 to $5 \mu \mathrm{m}$ (according to the manufacturer). This smoke is produced by the burning of the Ondina El Shell oil in a smoke generator.

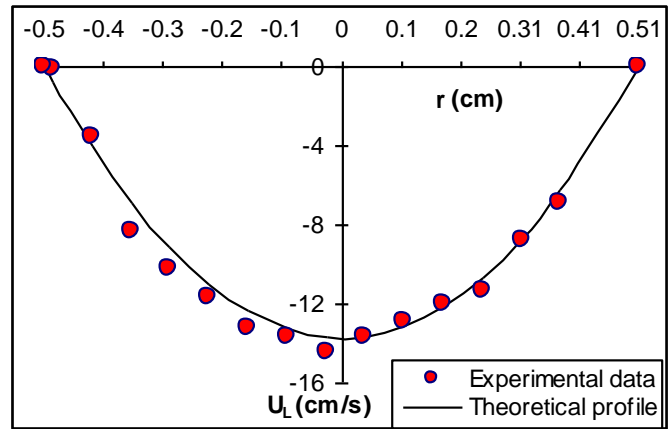

Fig. 2. Example of velocity profile in the liquid single phase flow

The qualification of the measurements by LDA, was made in the laminar liquid single phase flow. The comparison between the experimental data and the theoretical velocity profiles Fig. 2, agrees perfectly with less than $1 \%$. One can also reasonably estimates the radial position of the measuring point within $\pm 0.1 \mathrm{~mm}$, and the accuracy of the measurement of the velocity is about $1 \%$.

\subsubsection{Optical Method and Conductimetric Method}

Two methods have used to measure the film thickness, the optical and conductimetric methods. Thereafter, the thickness of liquid film will be evaluated by the optical method. The results obtained by the conductimetric method will be considered only qualitatively.

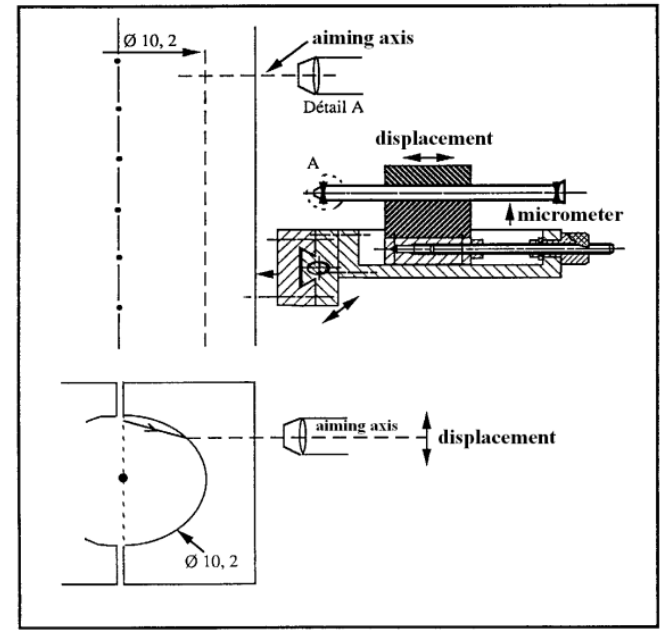

Fig. 3. The site and displacement of the optical glasses 'micrometer', cotes (mm).

The optical method Fig. 3 gives direct measurements of the film thickness. It makes an accuracy optical glass (micrometer eyepiece) which extending, and was made with the aid of glass blade graduated in thirtieth of millimetre. One division of the micrometer corresponds to $0.028 \mathrm{~mm}$. Since a division of the micrometer can easily be read for a clear image, an error of $\pm 3.5 \mu \mathrm{m}$ on the reading is acceptable.
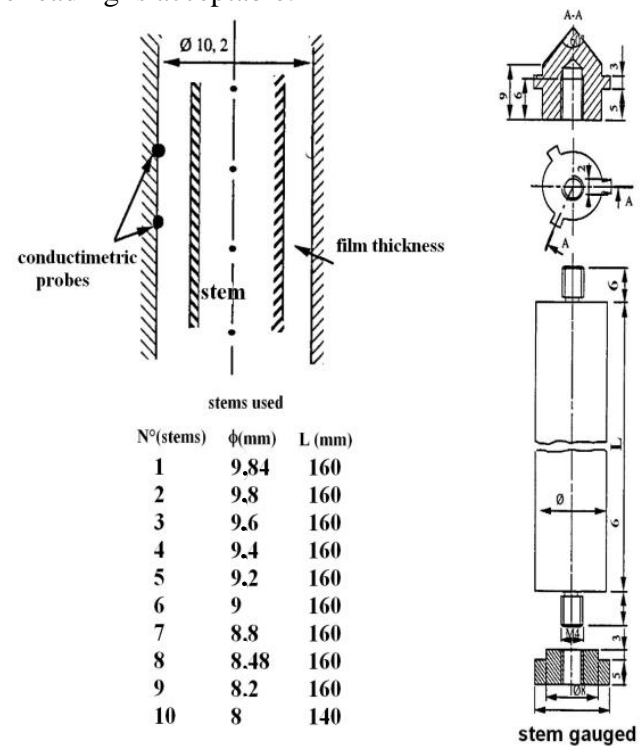

Fig. 4. Calibration proceedings of film thickness using the gauged stems for the two methods optical and conductimetric (dimensions in $\mathrm{mm}$ ).

To calibrate the micrometer eyepiece, different diameters of plexiglas rods which simulate different liquid film thicknesses were introduced slowly into the 
main measuring element Fig. 4. Then the numbers $\mathrm{N}$ of divisions of the micrometer corresponding to the thicknesses " $e$ " read. The rod diameters were measured with a micrometer with an accuracy of $\pm 0.005 \mathrm{~mm}$. These measurements are plotted in Fig. 6, and can be represented by the correlation: $N=32.14 e+18.21$, where the thickness $e$ is in $\mathrm{mm}$. Considering the nature and the geometry of our measurement element, we estimate that the distortion close to the wall remains unimportant and influences slightly the precision evaluated thickness.

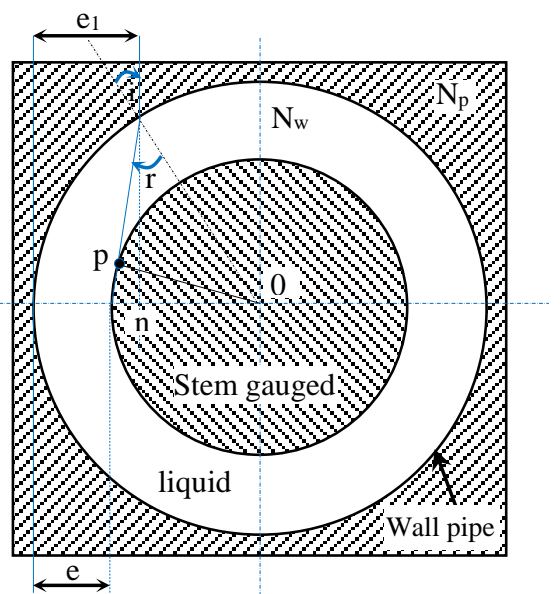

Fig. 5. Optical ways consideration for luminous rays

Considering the linearity of our calibration curve, a theoretical relation is determined between the liquid film thickness and the number of scales given by the micrometer. Relaying on the principle of Descartes and assuming that all rays coming from the object are perpendicular to the measuring element, see Fig. 5: we obtain: $e_{1}=R\left(1-N_{w} / N p\right)+\left(N_{w} / N_{p}\right) e$, Where: $e$ is the real thickness of liquid film, $e_{1}$ : thickness allotted by the micrometer, $N_{w}$ is the refraction index of liquid (1.33) and $N_{p}$ is the refraction index of Plexiglas. From this relation, the refraction index of the plexiglas can be estimated from our experimental values of " $e$ " and " $e_{1}$ ". This last is of $N_{p}=1.47$ to $\pm 1 \%$, value which close to manufacture 1.5 to $\pm 2 \%$. The theoretical relation can be translated in the form of the correlation equation by considering that one division of the micrometer is equal to $0.028 \mathrm{~mm}$.

Free falling rods were used in order to avoid effects of eccentricity. The edges of the rods have conical shapes and their lengths are sufficiently high to facilitate its centring in the measurement tube Fig. 4. With an accuracy of $\pm 0.005 \mathrm{~mm}$ for the measurements of the rod diameters, and of $\pm 0.03 \mathrm{~mm}$ for the diameter of the pipe, the liquid film thickness can be therefore determined with a precision of $\pm 0.03 \mathrm{~mm}$. It will be seen later that the thickness is about $0.35 \mathrm{~mm}$. Consequently, the relative errors on the measurements by this method can estimated less than $\pm 10 \%$ by taking the errors associated with the film thickness evaluation. All observations were made in the median plane of the tube normal to the viewing axis. In the micrometer field, the wall-liquid and the bubble-liquid interfaces appeared as sharp lines. Using a calibrated micrometer eyepiece the film thickness was readily measured.

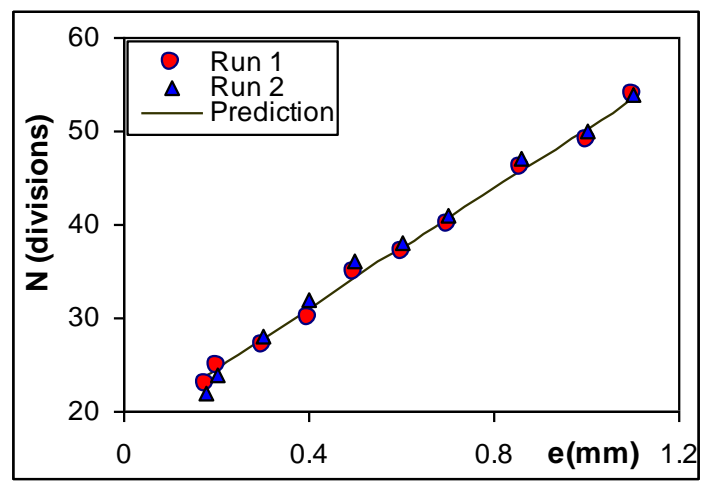

Fig. 6. Calibration: division number of micrometer $\mathrm{N}$ versus the film thickness "e".

The conductimetric method allows measuring the film thickness between the wall and the gas in a two-phase flow. It applies to liquids that are conductors of electricity, flowing on non conducting surfaces. We measure the conductance of a portion of film between the electrodes placed at the wall by applying a voltage between them. The relationship between the conductance ' $\mathrm{G}$ ' and the thickness ' $e$ ' is of the form $G / G_{\infty}=a-b \exp (-m e)$, where $\mathrm{a}, \mathrm{b}$ and $\mathrm{m}$ are constants determined experimentally and $G_{\infty}$ is the conductance of film in the limit of the infinite thickness. This measurement technique requires, first and foremost a choice of probes (geometries and electrodes) according to the liquid film thickness that one wishes to measure. In literature, different kinds of probes were used Collier and Hewitt (1967) and Coney (1973). For reason of facility of realization, circular electrodes were used whose theoretical and experimental study was conducted by $\mathrm{Yu}$ and Cognet (1986) interns' report of laboratory. This study determines the extension of the electric field and the influence of the geometry of the probe (diameter and spacing between probes) on the relationship between the conductance and the thickness. In our study the film thicknesses don't exceed one millimeter, we thus chose electrodes of diameter $\varnothing=1 \mathrm{~mm}$, separated by 3 $\mathrm{mm}$. These electrodes are located in the middle of the measuring element. These electrodes are powered by a conductimeter which provides an AC voltage in crenels.

The frequency is chosen large enough of $50 \mathrm{Khz}$ to make negligible the polarization phenomena. The calibration of this conductimeter was carried out using known carbon resistances. The results show that the relationship between the output voltage $\mathrm{V}$ and the conductance $\mathrm{G}$ is of the form $\mathrm{G} \sim \mathrm{aV}$. For the calibration of probes, we used interchangeable insulating plexiglas rods of various diameters (same ones used for the micrometer eyepiece calibration). The different diameters of rods simulate different thickness of liquid film. These stems are introduced into measuring element and the voltage $\mathrm{V}$ of conductimeter has been reported. The results of calibration shown that exponential shape is observed. We note that for the thickness below $0.5 \mathrm{~mm}$, the reproducibility of the 
results is not very good. Also, this method is used only qualitatively.

\subsubsection{The Taylor Bubble Velocity Measurements}

For the measurement of the bubble velocity, the bubble displacement time was measured with a stop watch over a known distance of $600 \mathrm{~mm}$. All measurements were taken sufficiently far from the injector, and were made from the top of the Taylor bubble. Each experiment was repeated a sufficient number of times in order to obtain a good average Dumitrescu-Taylor bubble velocity. It is expected that this average velocity is determined with an accuracy which remains less than $1 \%$.

\section{EXPERIMENTAL RESULTS AND DISCUSSION}

\subsection{Measurements of the Velocity Profile inside the Dumitrescu-Taylor Bubble}

The existing data on velocity profiles inside the Dumitrescu-Taylor bubble is very limited. The first attempts were made by Eccles (1972) and Rates (1972) using a hot wire anemometer. The Dumitrescu-Taylor bubble is held stationary by a counter-current flow in a $27 \mathrm{~mm}$ diameter tube. Their measurements were made in the vicinity of the Dumitrescu-Taylor bubble nose $(L / D<2.6)$, where $L_{b}$ is the bubble length and $D$ is a duct diameter.

In the present experiment, measurements of velocity profiles inside the Dumitrescu-Taylor bubble were made in the smooth zone at $150 \mathrm{~mm}$ below the bubble nose. The process consists in introducing a bubble of gas $\left(\mathrm{N}_{2}\right)$ mixed up with a small concentration of smoke, produced by the smoke generator. The intersection of two laser beams was located at the center pipe, where the maximum velocity is located. The symmetry of the setup makes it sufficient to determine the velocity profile only on the half of the pipe. The measurements were carried out in a short period of time to avoid the smoke particles dispersion. Thus, a new gas Dumitrescu Taylor bubble was introduced for each measured velocity profile. The results are illustrated in Fig. 7.

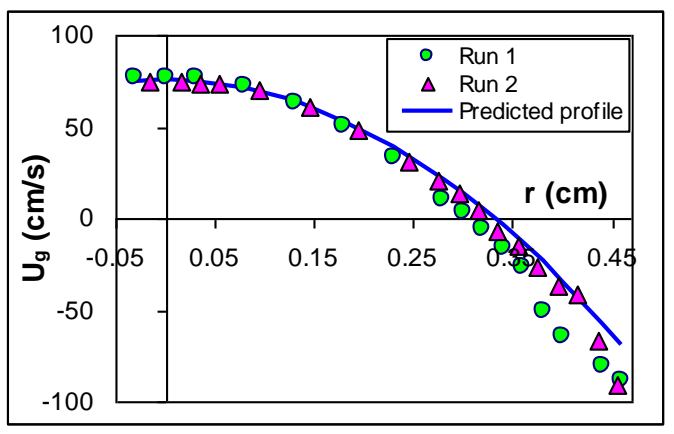

Fig. 7. Experimental data of gas velocity profiles obtained inside a Dumitrescu-Taylor bubble at $150 \mathrm{~mm}$ below the nose

Velocity values measured at the center pipe were more reproducible than those measured close to the interface. For consideration of the parabolic profiles, the optical correction of the radial position of the measurement point was also taken into account. This involves moving the central line by $0.3 \mathrm{~mm}$ for the first test, and $0.15 \mathrm{~mm}$ for the second one. The shape of the velocity profile is practically parabolic. Around the center, the velocity is upward, whereas it is downwards near the interface. This situation suggests the presence of a long toroïdal vortex inside the gas Dumitrescu-Taylor bubble. It should be pointed out that the previous work of Eccles (1972) reported by Filla (1976) does not show the existence of this vortex. Their measurements were carried out in the upper hemispherical portion of the bubble $(L / D<2.6)$ with a hot wire which doesn't enable to detect the flow direction. Also, the present measurements constitute to our knowledge, the first contribution to a better understanding of the kinematic within the Dumitrescu-Taylor bubble. The velocity value at the center of the pipe is $-76.7 \mathrm{~cm} / \mathrm{s}$, but is +80 to $+90 \mathrm{~cm} / \mathrm{s}$ at the interface. The difference between these two velocities may be due to the fact that the velocity profile is not perfectly parabolic. Comparison of the present results with those of Eccles (1972) shows the same form of velocity profile. He obtained a velocity of $49 \mathrm{~cm} / \mathrm{s}$ at the center compared to our value of $76.6 \mathrm{~cm} / \mathrm{s}$, and he reported 54 to $60 \mathrm{~cm} / \mathrm{s}$ at the interface while we measured 80 to $90 \mathrm{~cm} / \mathrm{s}$ in our case. The magnitude order of the wall shear stress on the gas side can be extrapolated from the slope of the gas velocity profile at the interface. It can be estimated at $\tau_{i g}=-0.013 \pm 0.003 \mathrm{~Pa}$.

From experimental velocity profiles obtained inside the bubble across a section, numerical integration leads to a flowrate which is almost zero:

$$
Q_{g}=\int 2 \pi r u(r) d r \approx 0
$$

The calculated velocity profile inside the gas, represented by continuous line (Fig. 7), shows a perfect agreement in the central part of the flow. However, disagreement becomes higher towards the interface.

From the physical properties of the liquid and the gas, the measured parameters of $\mathrm{U}_{\mathrm{gc}}=-75.7 \mathrm{~cm} / \mathrm{s}$ at $\pm 1 \%$, $e=0.0398 \pm 0.003 \mathrm{~cm}$ and the rising velocity $U_{T b}=0$, the calculated values of the velocity and the shear stress of the gas at the interface are about: $\mathrm{u}_{\mathrm{gi}}=75.7 \mathrm{~cm} / \mathrm{s}$ and $\tau_{g i}=-0.0112 \mathrm{~Pa}$. The relative errors between measured and extrapolated values of the gas shear stress at the interface are about $16 \%$. A magnitude order of the pressure gradient in the gas is also calculated $(-\partial \mathrm{P} / \partial \mathrm{z})$ $=-20$ Pa. $\mathrm{m}^{-1}$.

\subsection{Propagation Velocity of a Dumitrescu- Taylor Bubble}

In many branches of technology (distillation columns, nuclear reactor and oil-gas pipelines), and in vertical two-phase flow modeling, there is a need to know the rising velocity of the Dumitrescu-Taylor bubbles in liquids. For this reason, several theoretical and experimental studies have been investigated in vertical ducts to understand the mechanisms controlling this phenomenon (Dumitrescu 1943; Davies and Taylor 1950; White and Beardmore 1962; Zuber and Findlay 1965; Collins 1978; Nickens and Yannitell 1987; Orell 
and Rembrand 1986; Nicklin 1962; Griffith and Wallis 1961; Martin 1976; Mao and Dukler 1990; Van Hout et al. 2002). It seems that the velocity of the Taylor bubble in vertical co-current flow is reasonably understood. Recent works of Pinto et al. (2005) show that there are still investigations to bring. Especially for the evolution of the TB rise velocity when the liquid mode flow upstream of its nose passes from the laminar mode to the turbulent mode (the variations of the coefficient $\mathrm{C}_{2}$ Eq. (5)). In counter-current flow, the velocity remains difficult to be determined due to the bubble instability, as reported by Martin (1976). In the literature, for the downward flow, there are few measurements values of Dumitrescu-Taylor bubble velocity in vertical duct. This velocity measurement has been briefly reported by Nicklin (1962) and Griffith (1961), and has been investigated more extensively by Martin (1976). In our case, for all the range of used flow rates, the shape of the bubble doesn't change as the magnitude of the downward flow is increased (Fig. 8.d), which is not the case for the low diameter pipe $(26 \mathrm{~mm}$ ) used by Martin (1976) (Fig. 8.a, 8.b and 8.c). However, the purpose of our carried out measurements is to test if the empirical correlation for the Taylor bubble rise velocity in co-current flow can be applied in the case of counter-current flow.

The general form of the rise velocity of the elongated Dumitrescu-Taylor bubbles $U_{D T b}$ in upward (cocurrent) flow is expressed by equation:

$$
U_{D T b}=C_{1} \sqrt{g D}+C_{2} U_{\ell}
$$

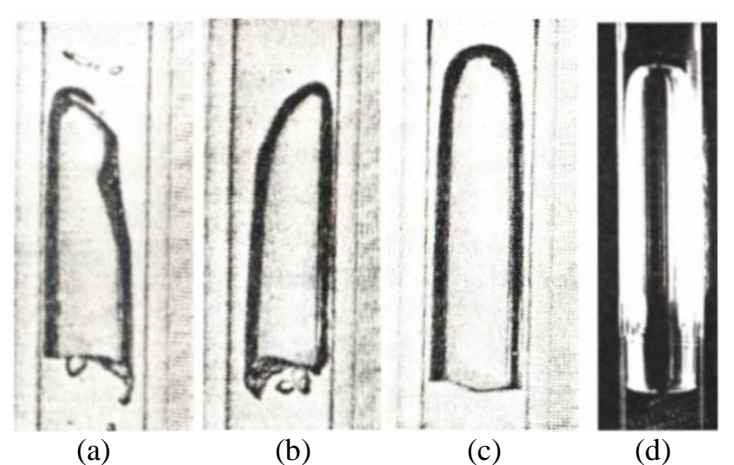

Fig. 8. Bubbles in $26 \mathrm{~mm}$ diameter pipe (a, b, c) and (d) $10.2 \mathrm{~mm}$ diameter pipe for various velocity flows

The first term in the right hand side of Eq. (1) represents the absolute translational velocity of the Dumitrescu-Taylor bubble in stagnant liquid $\left(U_{\ell}=0\right)$, where $g$ is the acceleration of gravity, $D$ is the pipe diameter and $U_{\ell}$ is the mean downward liquid velocity. The coefficient $C_{l}$ is a function of the physical properties of the liquid and the duct diameter. Thus, three meaningful dimensionless parameters are introduced by White and Beardmore (1962): the Froude number $F_{r}=U_{D T b} / \sqrt{g D}$ representative of the inertial effects (neglected if $F_{r}<0.0025$ ), the Eötvös number ( $E o=\rho_{l} g D^{2} / \sigma_{\ell}$ ) which represents the surface tension effects (negligible if, Eo > 70 and a Morton number $M o=g \mu_{\ell}^{4} / \rho_{\ell} \sigma_{\ell}^{3}$ containing mainly the properties of fluids (viscous effects are negligible for $M o<10^{-8}$ ). The value of $\mathrm{C}_{1}=0.35$ is admitted, provided that viscous and surface tension effects are negligible. This value has been accurately confirmed by experiments by numerous investigators (Nicklin 1962; Griffith and Wallis 1961; Dumitrescu 1943; White and Beardmore 1962; Nickens and Yannitell 1987). Otherwise, $C_{l}$ is graphically determined by White and Beardmore (1962). For the particular condition of the present work, the graph of White and Beardmore (1962), indicates that interfacial forces are important $E O=14.7$ and viscous forces are neglected $M_{o}=2.810^{-11}$.

The coefficient $C_{2}$ is about 1.2 according to Nicklin (1962) for a fully developed turbulent pipe flow. This value was interpreted by Collins (1978) as the ratio of the center line velocity of the liquid to the velocity of the liquid phase. In the laminar flow regime, the coefficient 1.2 is replaced by 2 , according to Collins et al. (1978). Our measurements are made in laminar flow (Re $\quad$ UL 2100$)$, where ReUL is the Reynolds number of the flowing liquid with mean velocity $\left(\mathrm{U}_{\ell}\right)$. The experimental data of the Taylor bubble velocity is plotted versus the mean value of the liquid velocity. This last is chosen to be negative because of the descending flow (Fig. 9). The Dumitrescu-Taylor bubble rising velocity is considered positive when it moves up and negative in the contrary.

Our results lead for $\operatorname{ReuL}<2100$, to:

$U_{D T b}=0,235 \sqrt{g D}+1,066 U_{\ell}$

Where $U_{\ell}$ is the liquid velocity flow, which is equal to $4 Q_{l} / \pi D^{2}$, where $Q_{\ell}$ is the liquid flowrate. $\operatorname{Re} U L$ is the Reynolds number of the flowing liquid with mean velocity. Figure 10 shows the zoom carried out from Fig. 9 for the laminar flow. According to Eq. (1), the results show a linear correlation between $U_{D T b}$ and $U_{\ell}$. The value of $C_{1}=0,235$ is in good agreement with the value determined from the graph for $E o=14,7$ White and Beardmore (1962). The value of the coefficient $C_{2}=1,066$ doesn't correspond to the calculated value Collins et al. (1978) ( $C_{2} \approx 2$ in the laminar flow). In Fig. 9, we also included the experimental results of Martin (1978), Nicklin (1962) for $D=26 \mathrm{~mm}$, and Griffith and Wallis (1961) for $D=12.7 \mathrm{~mm}$ at $t=10^{\circ} \mathrm{C}$. These results are obtained for the case of the displacement of Dumitrescu-Taylor bubbles in counter-current flow. Their measurements lead to the following expressions:

[Nicklin, $E o=90.23, M o=2.610^{-11}$ and $\operatorname{Re}_{\mathrm{ul}}<2100$ ]

$U_{D T b}=0.31 \sqrt{g D}+0.91 U_{\ell}$

[Griffith, $E o=21.32, M o=710^{-11}$ and $\operatorname{Re}_{U L}<2100$ ]

$U_{D T b}=0.30 \sqrt{g D}+0.25 U_{\ell}$ 
[Martin, Eo $=90.93, \quad M o=2.610^{-11}$ and for $\operatorname{Re}_{U L}<2100$ and $\left.\operatorname{Re}_{U L}>4000\right]$

$U_{D T b}=0.31 \sqrt{g D}+1 . U_{\ell}$

For Nicklin (1962), Griffth (1961), and Martin (1976), the experimental values of $C_{l}$ are in good agreement with the value in the graphical correlation (White and Beardmore 1962). The value of $C_{2}$ is $C_{2}=0.91$ (Nicklin), $C_{2} \approx 1$ (Martin). These values are close to our value. The Martin results don't show the apparent difference in the calculated $C_{2}$ value for the laminar and turbulent flow (estimated to $2 \%$ ). The slope of the straight lines mentioned on Fig. 9 represents the coefficient $C_{2}$. However, the $C_{2}$ of Griffith (1961) is equal to 0.25 . In his work, Griffith mentioned that it was difficult for him to stabilize the Dumitrescu-Taylor bubble when the liquid velocity downward flow increases. The bubble becomes alternately eccentric on one side or another, and to lean over one side of the pipe. These conditions may explain the value of $C_{2}$ which is far from than 1 . Therefore, we can say from our results obtained in laminar flow that in countercurrent flow, the equation form for the DumitrescuTaylor bubble velocity is preserved. On the other hand, the coefficient value $C_{2}$ of the Dumitrescu-Taylor bubble rise velocity in counter-current flow deviates strongly of that in co-current flow.

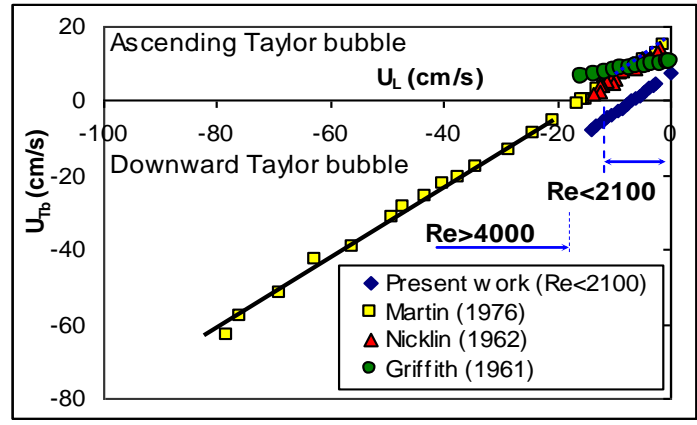

Fig. 9. Dumitrescu-Taylor Bubble velocity function of the mean liquid velocity

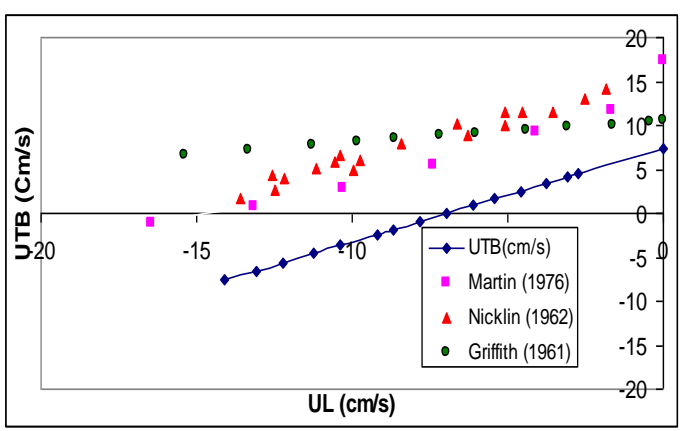

Fig. 10. Zoom brought back in the part of the bubble velocity at $\operatorname{Re}_{\mathrm{UL}}<2100$

\subsection{Erosion of the Dumitrescu-Taylor Bubble}

Nitrogen was bubbled into the liquid in order to have a saturated nitrogen solution, and thus avoiding mass transfer from the gas Dumitrescu-Taylor bubble to the liquid. Unfortunately, this was not the case because an erosion of the Taylor bubble with time was noticed.

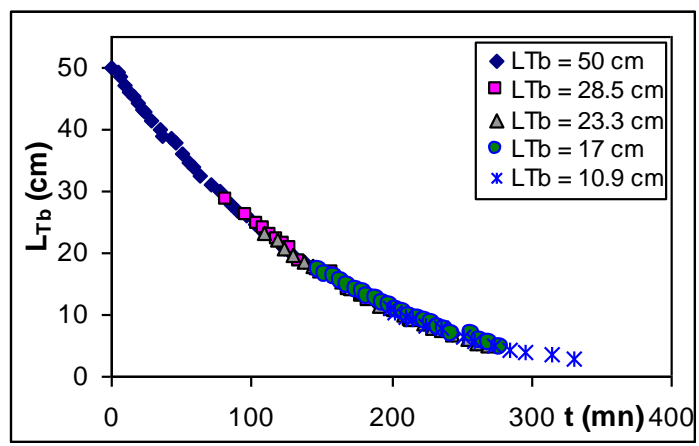

Fig. 11. Dumitrescu-Taylor bubble erosion versus exposure time for various length

The length of the bubble was measured every 5 minutes for different values of $L_{b}$, where $L_{b}$ represents the bubble length. An example is shown in Fig. 8. We notice, that with an adequate lag time, the decay curves are perfectly superimpose. This phenomenon of bubble decrease is complex. It is caused not only by one phenomenon such as the diffusion process for example Niranjan et al. (1988) but by a whole of variable physical phenomena induced by the flow around the bubble according to the length of the bubble (advection process).

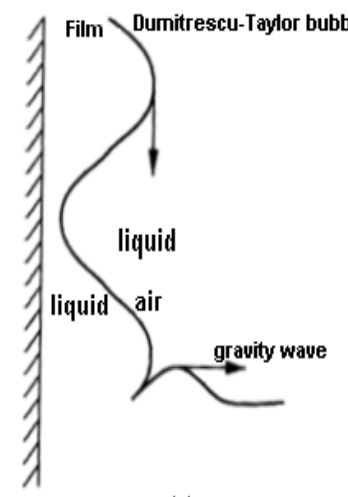

(a)

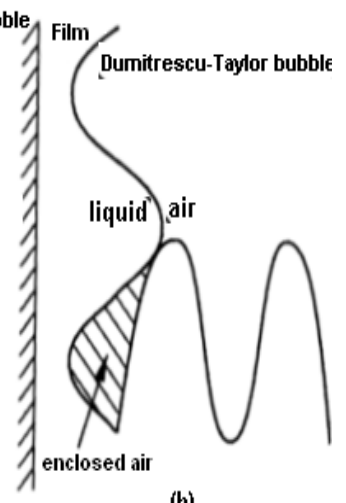

(b)
Fig. 12. Entrainment mechanism at the bottom of the Dumitrescu-Taylor bubble due to distortions on the falling liquid film for a long bubble Kockx, Delfos et

$$
\text { al. (2005) }
$$

Before, we describe what we have observed during the experiment. When the bubble is fixed up to $50 \mathrm{~cm}$ length see Fig. 17b, we have observed small gas bubbles which are torn off the bubble bottom. The amount of escaped gas in form of small gas is very significant. In this situation, the bubble erosion is due to distortion on the falling liquid film as described by Kockx, Delfos et al. (2005). In this situation, the gas entrainment process from the bubble is much more explanatory of the diagram Fig. 12 proposed by them. They explained that by: "when a wave on the film surface approaches the pool it generates a gravity wave, which propagates on the pool surface. When the slope of the induced gravity wave is large enough and the waves on the film surface travel fast enough, the crest 
of the gravity wave will contact the next wave on the film surface and air will be enclosed". Down to $50 \mathrm{~cm}$ length, the escaped gas which always results in small gas bubbles from the circumference of the base becomes weak, the bubble puts $7 \mathrm{mn}$ for decrease of 1.3 $\mathrm{cm}$ and the volume of this small gas bubbles are even smaller than quote before. On the other hand, when the bubble reached $L_{b}=22 \mathrm{~cm}$, the flow in the liquid film is entirely stable and the end of the bubble is almost not agitated, the decrease of Dumitrescu-Taylor bubble continues but more slowly, for example the bubble puts $18 \mathrm{mn}$ for decrease of $1.3 \mathrm{~cm}$. In this case, the bubble bottom are not loaded with small bubble, that means there exists a minimum film velocity below which no bubbles are produced (Fernandes et al. 1983). This description is the same when the bubble starts to decrease from $12 \mathrm{~cm}$ in length. Down to $5 \mathrm{~cm}$ length, the decrease of bubble is done but it continues even more slowly than that described previously, bubble puts $46 \mathrm{mn}$ for decrease of $1.3 \mathrm{~cm}$. We do not take the measures of the bubble erosion from $L_{b}=3 \mathrm{~cm}$. The decrease of length takes more time, the bubble can puts several hours to disappear and the idea to make an extrapolation of the experimental curve is not allowed because the presence of impurities which have been observed at the interface, especially on its surface side and around the surface bottom which modifies the determining way time of the bubble decrease.

Starting from these experimental observations, four zones in the development of the velocity profile in the liquid film can be proposed. In the first zone, we suppose that the liquid flow surrounding the Dumitrescu-Taylor bubble is assumed potential on $4 \mathrm{~cm}$ distance below the nose bubble, the calculated mean velocity liquid film is approximately $30 \mathrm{~cm} / \mathrm{s}$. After this distance, the liquid film enters in the transition phase on distance of $9 \mathrm{~cm}$, the calculated mean liquid velocity is about $38 \mathrm{~cm} / \mathrm{s}$. Immediately after this distance the liquid flow reaches its terminal velocity (developed laminar flow) on the distance of $10 \mathrm{~cm}$ (i.e. $22 \mathrm{~cm}$ below the bubble nose), the calculated mean liquid film velocity $U_{\ell}$ is about $47 \mathrm{~cm} / \mathrm{s}$. From this distance, oscillations start to appear and become very accentuated beyond $L_{b}=25 \mathrm{~cm}$. In this region, the calculated mean liquid film is about $61 \mathrm{~cm}$. These velocities have been estimated from conservation of mass, i.e. the downward liquid flow rate above the Taylor bubble is equal to the liquid flow rate in the falling film at any location $\mathrm{z}$ downstream of the Dumitrescu-Taylor bubble nose as defined:

$Q_{\ell}=\frac{\pi}{4} D^{2} U_{\ell}=\frac{\pi}{4}\left(D^{2}-[D-2 e(z)]^{2}\right) U_{\ell f}(z)$

where $U_{\ell}$ is the downward liquid velocity above the stationary bubble, $U_{\ell f}$ the mean velocity liquid film, $e(z)$ is the film liquid thickness. Velocities then, increase with decreasing film thickness see Fig. 18 (or equivalently increasing the length bubble as measured by Kawaji and Ahmed (1997)). For this range of bubble length, we think that the dynamic of the bubble wake is different (the eye of the vortex, quite close to the bottom interface circulation and renewal of the wake)
From these experimental results, we have tried to estimate a velocity of mass transfer using the following procedure. From the experimental data of erosion bubble Fig. 11, when we divide the experimental curve of erosion bubble according to the four different regions along the bubble, the plotted values of length against time give graphs of straight-lines Fig. 13.
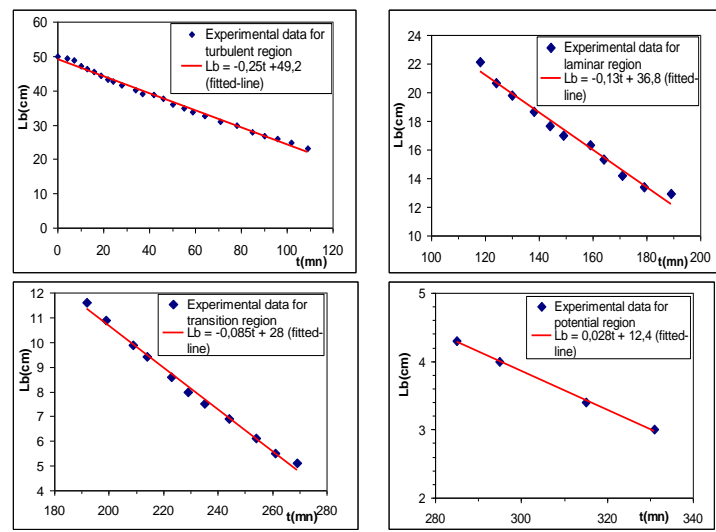

Fig. 13. Slopes given by the fitted-lines of decrease $\mathrm{L}_{b}(\mathrm{~cm})$ against $\mathrm{t}(\mathrm{mn})$ for different regions defined along bubble.

The slope of straight-line graphs has dimension as velocity $(d l / d t(\mathrm{~cm} / \mathrm{mn}))$. Then, we discuss our results from these slopes which represent a velocity of escaped gas from the bubble. It resembles to the coefficient of mass transfer. Figures 13a, 13b, 13c and $13 \mathrm{~d}$ show the different values of slope for the different regions along the bubble. The deduced values for slopes coefficient are about:

$v_{0} \approx k_{L O}=4.7710^{-4} \mathrm{~cm} / \mathrm{s}$ for potential flow region, $v_{1} \approx k_{L 1}=1.4010^{-3} \mathrm{~cm} / \mathrm{s}$ for the transition region, $v_{2} \approx k_{L 2}=2.210^{-3} \mathrm{~cm} / \mathrm{s}$ in the laminar region and $v_{3} \approx k_{L 3}=4.210^{-3} \mathrm{~cm} / \mathrm{s}$ in the turbulent region.

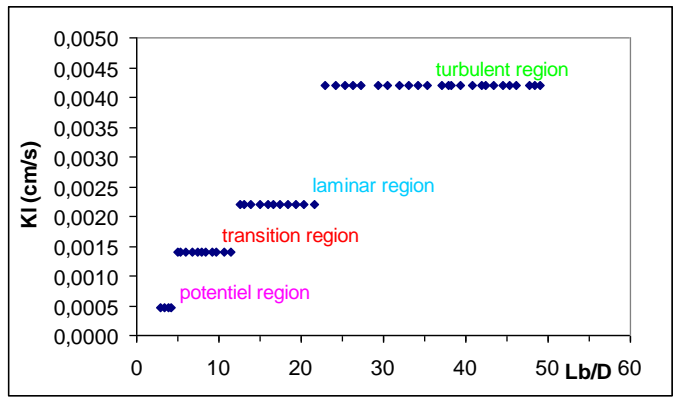

Fig. 14. Predicted mass transfer (average slopes distribution) from bubble at different regions defined along bubble length.

These values of slopes in Fig.14 show that erosion of bubble (decrease of its length) doesn't do in the same proportion according to these regions of bubble but in one region the escape gas remain constant as long as the hydrodynamic of the liquid film does not change Niranjan et al. (1988). From this graph Fig. 14, the erosion of bubble seems to increase linearly with $L_{b}$. We think that it is at the bottom bubble level where the 
prevalent mass transfer is carried out because the velocity at the surface bottom is not the same along the bubble and consequently the greater liquid film velocity at entry in to the wake would give rise to faster growth of instability which generates a complicated turbulent mixing process in the wake flow. Intuitively, since liquid film velocities are greater for longer DumitrescuTaylor bubble, we expected the mass transfer coefficient to increase with the Dumitrescu-Taylor length.

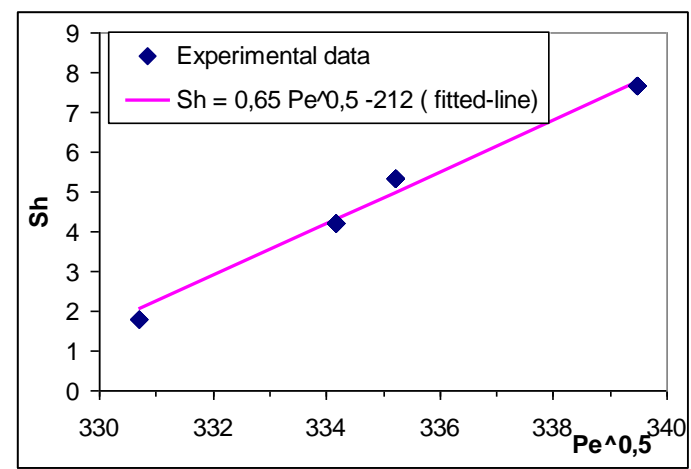

Fig. 15. The data plotted in Sh with $(\mathrm{Pe})^{1 / 2}$.

For this purpose as shown in Fig. 15, the data were correlated in terms of the Sherwood number $S h=k_{L} e / D$ and the Pecklet number $P_{e}=U_{l f} e / D$, where calculated by using the estimed values of velocity of escaped gas from the bubble $\left(v \approx k_{L}\right)$ and $U_{\text {lf }}$ mean velocity of liquid film and $e$ film thickness in each region. $D=1.6510^{-5} \mathrm{~cm} / \mathrm{s}^{2}$ : being the liquid-phase diffusion coefficient. Then, the line which fits the data gives $S h=0.65 P_{e}^{0,5}-212$.

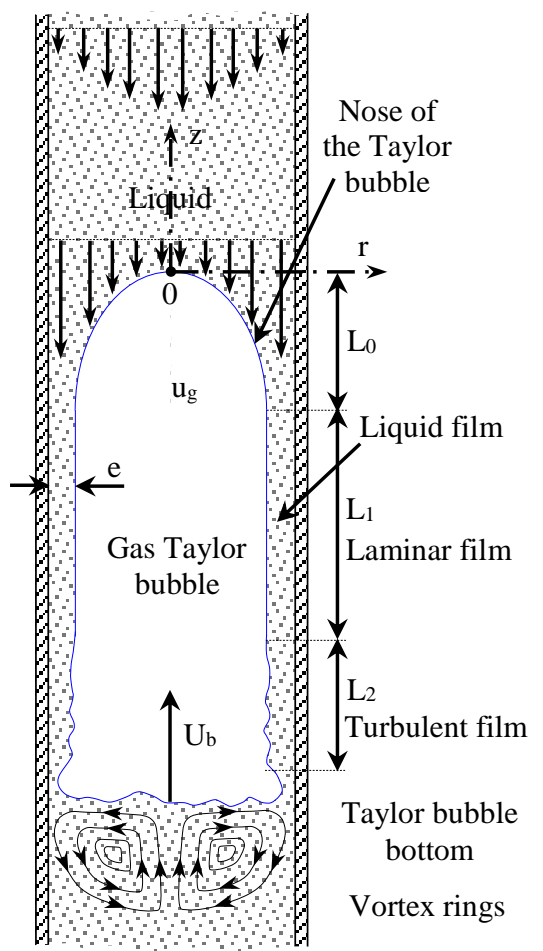

Fig. 16. Schematic representation of the flow around a Dumitrescu-Taylor bubble

\subsection{Visuals Observations}

The global geometrical shape of the Taylor bubble was first observed with naked eyes. These observations of a fixed gas bubble with a length $L_{D T b}$ above $30 \mathrm{~cm}$, helped to distinguish three different regions (Fig. 16).

The entrance region with length $L_{o}$ is of about 4 to $50 \mathrm{~mm}$ (5D). This region consists of two types of flow, an irrotationnal flow on a distance of $2,6 \mathrm{~mm}(\mathrm{D} / 4)$ below the nose of the bubble Dumitriscu (1943), and a complex flow corresponding to a transitional flow (Brown 1965).

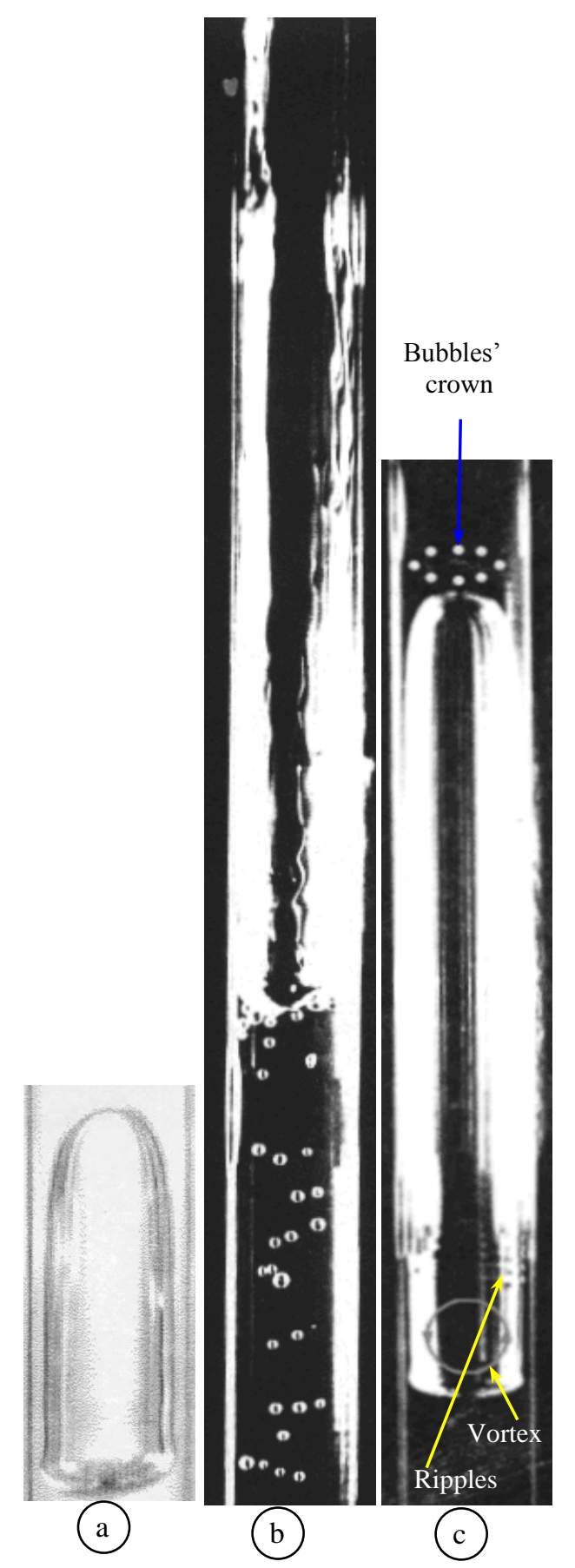

Fig. 17. Pictures taken for various lengths of the Dumitrescu-Taylor bubble: a) $\mathrm{L}_{\mathrm{DTb}}=2.2 \mathrm{~cm}$; b) Disturbed region where $\left.\mathrm{L}_{\mathrm{DTb}}=80 \mathrm{~cm} ; \mathrm{c}\right) \mathrm{L}_{\mathrm{DTb}}=6 \mathrm{~cm}$. 
A second region of a length $L_{1}$ about $18 \mathrm{~cm}$ (18D) is similar to free laminar flow along a cylinder (laminar film). The third region has a length $L_{2}$ which depends on the bubble being studied. It corresponds to the part of the film disturbed. These disturbances appear beginning from $L_{D T b}=22 \pm 1 \mathrm{~cm}$. They start by small disturbances of the liquid film along distances of less than $1 \mathrm{~cm}$. Further away, the film becomes unstable and similar to a turbulent film. These distances were confirmed during the examination of the signals obtained relating to the thickness of liquid film by the conductimetric method. The evolution of the liquid film thickness (Fig. 18), shows well the lengths characteristic of the areas $L_{o}, L_{1}$ and $L_{2}$.

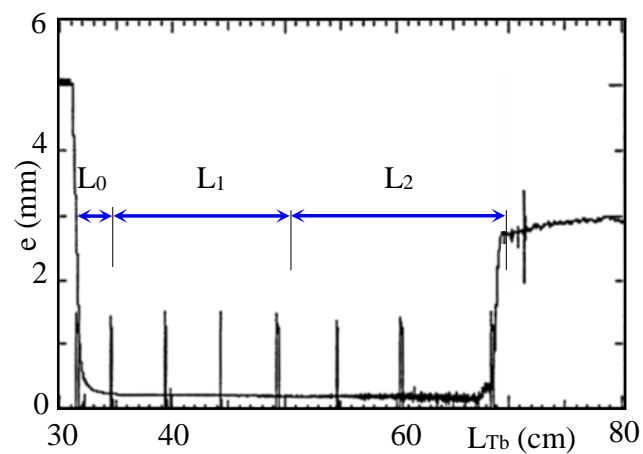

Fig. 18. Evolution of the liquid film thickness along the Dumitrescu-Taylor bubble of length $\mathrm{L}_{b}=38 \mathrm{~cm}$.

On this figure, some pulses were recorded. The first corresponds to the passage of the Taylor bubble nose, and the last to his bottom. The others intermediate pulses were recorded at every $5 \mathrm{~cm}$, and serve as indicators of the position along the bubble. It can be observed that on the area $L_{1}$ of the order $18 D$, a very light variation of thickness in the film is presumably laminar. We consider that the flow becomes fully developed from the distance of 10 to $12 \mathrm{D}$ below the nose of the bubble.

This distance was estimated theoretically by Campos and Guedes de Carvalho (1988), by the following expression:

$L_{f d}=\frac{\left(\frac{g e_{f}^{2}}{2 v_{\ell}}+U_{D T b}\right)^{2}}{2 g}$

Where $e_{f}$ is the thickness of the liquid film, and $U_{D T b}$ is the bubble velocity. The calculated distance from this Eq. (6) is of the order $3 D$, value which is much lower than the experimental one, where $D$ is the duct diameter. This gap is probably due to the assumption of non-viscous flow during the establishment of the Eq. (6). We have calculated this distance by taking the value of the film thickness obtained by the optical method. The values of the film thickness obtained by the conductimetric method were not retained, because in the range from the thicknesses to be measured, the results of calibration are less reproducible. Beyond $L_{l}$, significant fluctuations appear on the film thickness signal. This region will be regarded as a turbulent film zone.
For bubble lengths lower than $21 \mathrm{~cm}$ and larger than 3 $\mathrm{cm}$, we noted the formation of the circular ripples on the lower part of the Taylor bubble (Fig. 17c). The number of these ripples is 7 or 8 . The first two ripples are not very visible compared with the others which are following. So, the amplitude of these ripples tends to increase gradually from the first ripples to the last. With the eye naked, the five last ripples seem to have the same amplitude and the last ripple seem also to be located always at a determined same distance ahead of the Dumitrescu-Taylor bubble bottom. Let us note that the visualization of these ripples requires a good lighting with a good contrast in light. For various lengths of Dumitrescu-Taylor bubbles, images were taken by an analog fast camera (Kodak Ektopro 1000) and analyzed. An example of photographs is given on the Figs. 19 and 20). From these images, the wavelength of ripples and the distance from which the last ripple is located from the rear bottom of the bubble are determined.

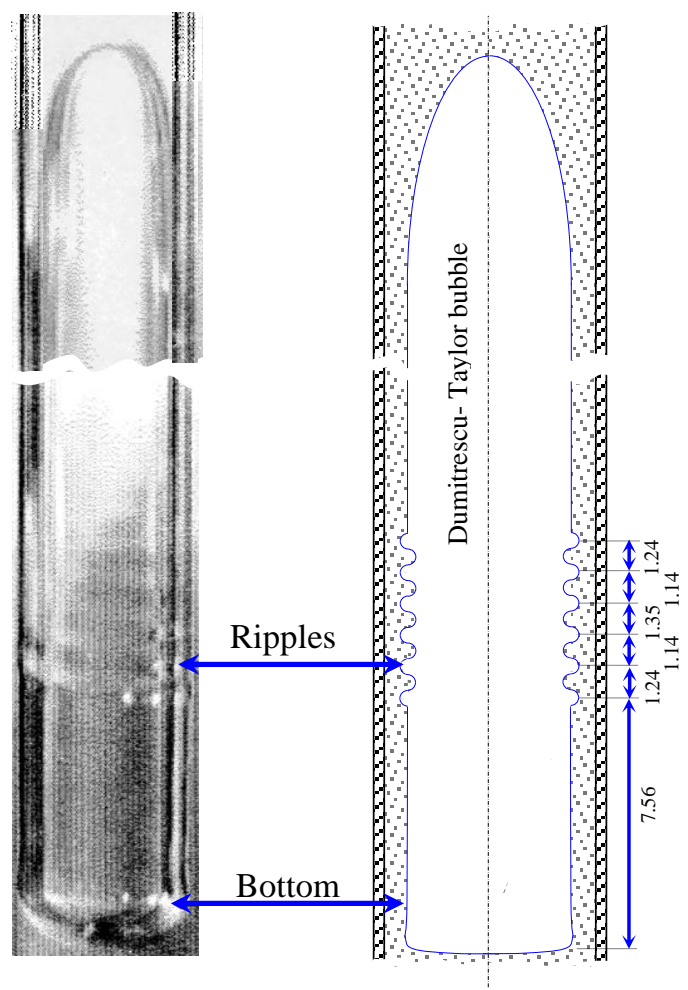

Fig. 19. Wavelengths values measured and the distance separating the first ripple from the bottom (in $\mathrm{mm}$ ):

Dumitrescu-Taylor bubble of length $5 \mathrm{~cm}$.

We schematized the results of measurements concerning these ripples beside each image (Fig.19 and Fig. 20). We indicate the wavelengths $\lambda$ and the located distance of the last ripple up the rear bottom of the bubble. As one can note it, these ripples or "undulations" seem to have the same wavelength of about $1.24 \mathrm{~mm}$. As for the located distance of the last ripple from the rear bottom bubble, it is $0.8 \mathrm{~cm}$ approximately and seems to be independent of the Dumitrescu-Taylor bubble length. The ripples observed are quite stationary and appear only for DumitrescuTaylor bubble lengths superiors to $3 \mathrm{~cm}$ (Fig. 10a). Let 
us note that this type of ripples has observed and analytically described by Bretherton (1961).

These are also observed in experimental works of Van Heuven, Beek (1963) and Nigmatulin and Bonetto (1977). For Van Heuven and Beek, the wavelengths measured are from 1.3 to $2 \mathrm{~mm}$ for lengths of Taylor bubbles from 1.5 to $1 \mathrm{~cm}$ in tubes of diameter of $2.4 \mathrm{~mm}$ and $4.8 \mathrm{~mm}$. They observed only two ripples just formed below the Dumitrescu-Taylor bubble bottom.

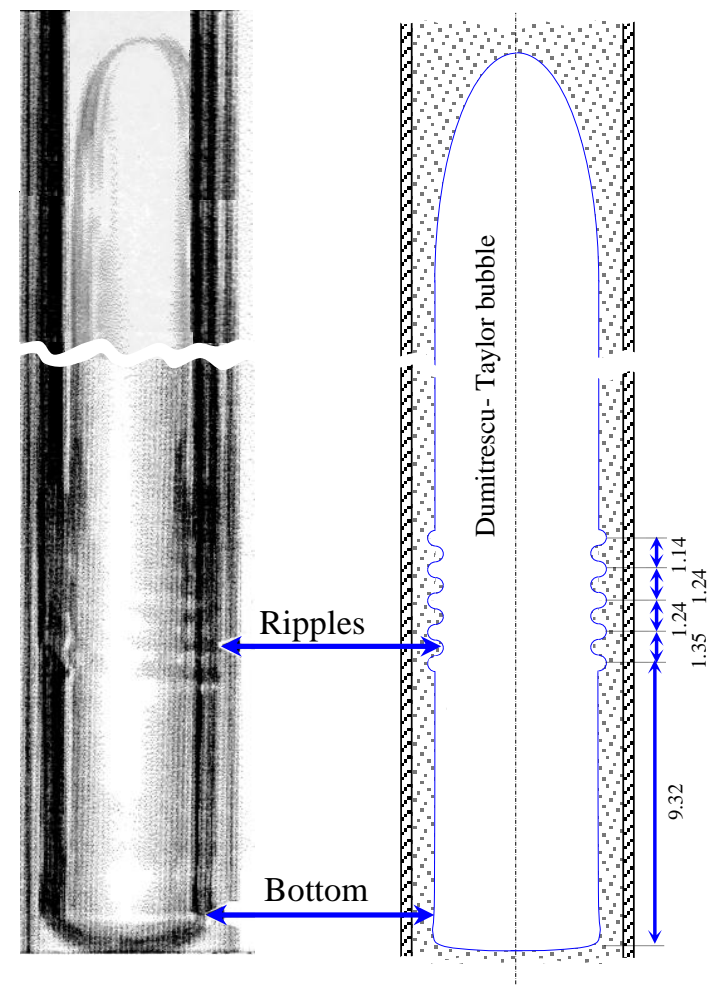

Fig. 20. Wavelengths values measured and the distance separating the first ripple from the bottom (in $\mathrm{mm}$ ): Dumitrescu-Taylor bubble of length $8 \mathrm{~cm}$

The works of Nigmatulin and Bonetto (1977) on the Dumitrescu-Taylor bubble were done in $15.6 \mathrm{~mm}$ diameter of vertical glass tube, and which mention the presence of these ripples. They find that the amplitudes of the ripples were inverse proportional to the length of the Dumitrescu-Taylor bubble, and for the long bubble they were visible only near the bottom of the bubble. The presence of these ripples on the lower part of the Dumitrescu-Taylor bubble seems to be due to the existence of a gradient of surface tension in bottom of the bubble Ellis (1966), Otto (1973). For a flow on free plan, the wavelengths $\lambda$ of the ripples are related to the celerity of propagation of these same ripples Lamb (1959) by:

$\lambda=\frac{2 \pi \sigma}{\rho_{\ell} U_{\ell}^{2}}$

Where $U_{\ell}$ is the liquid velocity at the interface of the ripples. The flow of the liquid film making stationary the bubble is $Q_{f}=5.510^{-6} \pm 1 \% \mathrm{~m}^{3} / \mathrm{s}$. Under these conditions, the theory of Nusselt, if the velocity at the interface is of $70 \mathrm{~cm} / \mathrm{s}$, leads to a value of the wavelength $\lambda=0.92 \mathrm{~mm}$, which is of the same order of magnitude as the measured value. It will be noted that if $U_{\ell}$ is taken as mean velocity in the film $(47 \mathrm{~cm} / \mathrm{s}$ approximately), one will find a value of $\lambda$ of $2 \mathrm{~mm}$. We note that this description is related to an established flat profile, which is not the case of our situation. That's why, the values of $\lambda$ are situated between 0.9 and $2 \mathrm{~mm}$.

New and interesting phenomena are observed primarily ahead of the Dumitrescu-Taylor bubble nose and on the side of its end. First observation is the formation of crown of small bubbles ahead of the Dumitrescu-Taylor bubble nose. When this latter is taken stationary, we observe small bubbles less than one millimetre of diameter, that's coming from the circuit to take place at a distance of $5 \mathrm{~mm}$ ahead the nose of bubble and from a radial position at about $1 \mathrm{~mm}$ (Fig. 17c). They formed a crown around the Taylor bubble nose. This crown is composed of 8 to 9 equidistanted small bubbles. It remains stable even while making move the bubble at very low liquid flowrates. Our observations show that all the small bubbles have practically the same diameter. In fact, when another very small bubble takes place in the crown, this last can be driven out of its place, and replaced by another bubble which has an adequate diameter (about $1 \mathrm{~mm}$ ). We have tried from the force balances applied to small bubble (Hervieu 1988) to determine the range of the bubbles diameters which could to position on this circumference above the nose Dumitrescu-Taylor bubble.

We have obtained weak bubble diameters compared to experimental found diameter. Probably, this great variation comes from the uniform velocity profile assumed for the calculated facility We mention that these bubbles come each one individual and those which go down on a trajectory close to the wall to approximately $1 \mathrm{~mm}$ are favourably wedged in this zone and the crown of small bubble was observed only with the presence of the fixed bubble. These observations were not described in the literature. It was shown in literature (Segré and Silberberg 1962), that in laminar flow of suspension of spheres through a cylindrical pipe, spheres are displaced radially outwards from the centre and inwards from the wall, and that a stationary build up of concentration occurs at a radial position at about 0.6 tube radii from the axis and the distribution for large and small spheres differ considerably from each other.

The other phenomenon observed is the formation of vortex on the lateral side at the end of the Taylor bubble. In Fig. 17c, we represented the vortex considering only the front face. The second vortex is localized in the opposite side, and the rotation of each one is in the reverse clockwise direction. These vortices are very visible on the two parallel external faces of the measured transparent element. On the other hand, on the two parallel other parallel side faces (not very transparent), the observation is difficult. Probably, we imagine the existence of two others vortex at the level of the side faces by symmetry. We have described only what we observed because photography was difficult to take. 


\section{Conclusion}

The experimental study has been done on the Dumitrescu-Taylor bubble in a downward laminar counter-current flow in a smooth vertical duct. For low diameter duct (low Eötvös value number), the Dumitrescu-Taylor bubble shape is more preserved and the bubble can be stabilized without difficult.Our results include:

- The shape of measured velocity profile inside the Dumitrescu-Taylor bubble by Laser Doppler Velocimetry (LDA) in laminar zone is practically parabolic. These measurements confirm the presence of a long toroïdal vortex. A comparison between experimental velocity profiles and the calculated one, show a good agreement. In addition, an order of magnitude of the pressure gradient in gas has been estimated. The measurements data of gas velocities inside the Dumitrescu-Taylor bubble constitute to our knowledge, the first contribution to a better understanding of the kinematics within the Taylor bubble.

- The rise bubble velocity results were presented in counter-current flow. For this configuration study, only few experimental works are available in the literature. The Dumitrescu-Taylor bubble velocity equation is similar to that of the Dumitrescu-Taylor bubble rising in the co-current flows. The parameters $C_{1}$ and $C_{2}$ in the velocity equation are determined experimentally, and compared with those reported by some authors.

- The experimental measurements obtained on the erosion of the Taylor bubble are described and detailed. It can be deduced that erosion of bubble (decrease of its length) cannot be in the same proportion along its axis, but in one region of this bubble the escape gas remains constant as long as the hydrodynamics of the liquid film does not change. It was found that this mass transfer of gas increases with the Dumitrescu-Taylor length.

- The presence of the circular ripples ahead the Dumitrescu-Taylor bubble bottom seems to be quite stationary, and this leads to be said that these ripples constitute a natural phenomenon which always accompanies the flow around the bubble.

\section{REFERENCES}

Adeney, W.E. and H.G. Becker (1919). The determination of the rate of solution of atmospheric nitrogen and oxygen by water. Phil. Mag. S. 6, 38(225), 317-337.

Baker, O. (1954). Simultaneous flow of oil and gas. Oil and Gas J. 53, 185-195.

Bendiksen, K. (1985). On the motion of long bubbles in vertical tubes. Int. J. Multiphase Flow 11(6), 797812.

Bretherton, F.P. (1961). The motion of long bubbles in tubes. J. Fluid Mech. 10, 166-187.
Brown, R. A. S., (1965). The mechanics of large gas bubbles in tubes I. Bubble velocities in stagnant liquids. Can. J. Chem. Eng., Vol. 43, pp. 217-223.

Bugg, J.D., K. Mack and K.S. Rezkallah (1998). A numerical model of Taylor bubbles rising through stagnant liquids in vertical tubes. Int. J. Multiphase Flow 25(2), 271-281.

Clanet, C., P. Héraud and G. Searby (2004). On the motion of bubbles in vertical tubes of arbitrary cross-sections: some complements to the Dumitrescu-Taylor problem. J. Fluid Mech. 519, 359-376.

Collier, J.G. and G.F. Hewitt (1967). Film thickness measurement in two phase flow. British Chemi. Eng. 12(5), 705-715.

Collins, R. (1978). The motion of a large gas bubble rising through liquid flowing in a tube. J. Fluid Mech. 89, 497-514.

Coney, M.W.E. (1973). The theory and application of conductance probes for the measurement of liquid film thickness in two phase flow. J. Phys. E: Sc. Instr. 6, 903-910

Campos, J.B.L.M. and J.R.F. Guedes de Carvalho (1988a). An expérimental study of the wake of gaz slugs rising in liquids. J. Fluid Mech. 196, 27-37.

Davies, R. M. And G.I. Taylor (1950). The mechanics of large bubbles rising through extended liquids and through liquids in tubes. Proc. R. Soc., London Ser. A $200,375-390$.

Dumitrescu, D. T., (1943). Strömung an einer Luftblase in senkrechten Rohr. Z. Angew. Math. Mech., Vol. 23, pp.139-149.

Durrani, T.S. and C.A. Greated (1977). Laser Systems in Flow Measurement. Plenum Press, New York.

Durst, F., A. Melling and J.H. Whitelaw (1976). Principales and practices of Laser Doppler Anemometry. Academic Press, New York.

Eccles, M.A. (1972). Research Project Report. Chem. Eng. Department, University of Cambridge, England.

Ellis, S.R.M. and M.W. Biddulph (1967). The effect of surface tension characteristics on plate efficiencies. Chem. Engrs. Reseach and Design 45, 223-228.

Fernandes, R.D., R. Semiat and A.E. Dukler (1983). Hydrodynamic modele for gas-liquid slug low in vertical tubes. Aiche J. 29(6), 981-989.

Filla, M.J., J.F. Davidson, J.F. Bates and M.A. Eccles (1976). Gas phase controlled mass transfer from a bubble. Chem. Sc. 31, 359-367.

Goldsmith, H.L. and S.G. Mason (1962). The movement of single large bubbles in closed vertical tubes. J. Fluid. Mech. 14, 42-58. 
Griffith, P. And G.B. Wallis (1961). Two-phase slug flow. J. of Heat Transfer, Trans. ASME, Serie C 83, 307-320.

Hervieu, E. (1988). Écoulements Monophasique et Diphasique dans un Branchement en TE: Étude Théorique et Expérimentale. Ph.D. Thesis, INPG, Grenoble, France.

Higbie, R. (1935). The rate of absorption of a pure gas into a still liquid during a short period of exposure. Trans. A.I.Ch. E. 31, 365-389.

Kawaji, M., J.M. De Jesus and G. Tudsoe (1997). Investigation of flow structures in vertical slug flow. Nucl. Eng. Des. 175, 37-48.

Kockx, J.P., F.T.M. Nieuwstadt, R.V.A. Oliemans and R. Delfos (2005). Gas entrainment by a liquid film falling around a stationary Taylor bubble in a vertical tube. International Journal of Multiphase Flow 31, 1-24.

Lamb, H. (1959). Hydrodynamics. Cambridge University Press.

Mandhane, J.M, G.A. Gregory and K. Aziz (1974). A flow pattern map for gas-liquid flow in Horizontal pipe. Int. J. Multiphase Flow 1, 537-553.

Martin, C. S. (1976). Vertically downward two-phase slug flow. J. of Fluids Eng. 98, 715-722.

Mao, Z. S. And A. Dukler (1990). The motion of Taylor bubbles in vertical tubes: I. A numerical simulation for the shape and rise velocity of Taylor bubbles in stagnant and flowing liquid. J. Comp. Phys. 91, 132-160.

Nicklin, D.J., J.O. Wilkes and J.F. Davidson (1962). Two-phase flow in vertical tubes. Trans. Inst. Chem. Eng. 40, 61-68.

Nickens, H.V. and D.W. Yannitell (1987). The effect of surface tension and viscosity on the rise velocity of a large gas bubble in a closed vertical liquid-filled tube. Int. J. Multiphase Flow 13, 57-69.

Niranjan, K., M.A. Hashim, A.B. Pandit and J.F. Davidson (1988). Liquid-Phase controlled mass transfer from a gas slug. Chem. Eng. Sci. 43(6), $1247-1252$.

Nogueira, S., M.L. Riethmuller, J.B.L.M. Campos and A.M.F.R. Pinto (2006). Flow in the nose region and annular film around a Taylor bubble rising through vertical columns of stagnant and flowing Newtonian liquids. Chemical Eng. Sci. 61, 845857.

Orell, A. and R. Rembrand (1986). A model for gasliquid slug flow in vertical tube. Ind. Eng. Chem. Fundam. 25(2), 196-206.
Pinto, A.M.F.R. and J.B.L.M. Campos (1996). Coalescence of two gas slugs rising in vertical column of liquid. Chem. Eng. Sci. 1(1), 45-54.

Pinto, A.M.F.R., M.N. Coelho Pinheiro, S. Nogueira, V.D. Ferreira and J.B.L.M. Campos (2005). Experimental Study on the Transition in the Velocity of Individual Taylor Bubbles in Vertical Upward Co-Current Liquid Flow. Trans IChemE, Part A, Chemical Engineering Research and Design 83(A9), 1103-1110.

Polonsky, S., L. Shemer and D. Barnea (1999). The relation between the Taylor bubble motion and the velocity field ahead of it. Int. J. Multiphase Flow 25, 957-975.

Rates, J.F. (1972). Research Project Report. Chem. Eng. Department, University of Cambridge, England.

Segré, G. and A. Silberberg (1962). Behaviour of macroscopic rigid spheres in Poiseuille flow. Part 1. Determination of local concentration by statistical analysis of particle passages through crossed light beams. Journal of Fluid Journal of Fluid Mechanics 14(1), 115-135.

Segré, G. and A. Silberberg (1962). Behaviour of macroscopic rigid spheres in Poiseuille flow. Part2. Experimental results and interpretation. Journal of Fluid Journal of Fluid Mechanics 14(1), 116-157.

Van Heuven, J.W. and W.J. Beek (1963). Gas absorption in narrow gas lifts. J. Chem. Eng. Sc. $18,377-390$

Van Hout, R., A. Gulitski, D. Barnea and L. Shemer (2002). Experimental investigation of the velocity field induced by a Taylor bubble rising in stagnant water. Int. J. Multiphase Flow 28, 579-596.

White, E.T. and R.H. Beardmore (1962). The velocity of rise of single cylindrical air bubbles through liquids contained in vertical tubes. Chem. Eng. Sci. 17, 351-361.

YU, Q. and G. Cognet (1984). Conductive probes for the liquid film thickness measurements. Report interne, LEMTA-INPL.

Zuber, N. And J.A. Findlay (1965). Average volumetric concentration in Two-Phase Systems. J. Heat Transfer, Trans. ASME, Serie C 87, 453-468. 